\title{
SUL COLLEGAMENTO DI UNA FUNZIONE DATA NEI PUNTI DI UNA SUPERFICIE CON UNA FUNZIONE DEI PUNTI DELLO SPAZIO, E SUA APPLICAZIONE ALLA TEORIA DELLA FUNZIONE POTENZIALE DI DOPPIO ST'RATO.
}

\section{GIAN ANTONIO MAGGI.}

La dimostrazione dell'esistenza e la ricerca delle proprietà delle derivate prime della funzione potenziale di doppio strato

$$
\varphi=\int_{\sigma} k \frac{\partial \frac{1}{r}}{\partial n} d \sigma,
$$

dove i simboli hanno il consueto significato, si riconduce facilmente a quella delle derivate prime della funzione potenziale di semplice strato, nell'ipotesi che la funzione $k$ sia derivabile, nei punti della superficie, rispetto alle coordinate cartesiane $a, b, c$ degli stessi punti. E quanto io faccio nella mia antica Memoria sulla Funzione Potenziale, pubblicata in questo stesso giornale 1). Mi valgo ivi di un risultato di Beltrami, il quale però non rappresenta per lui che un termine di passaggio, per giungere ad una formula informata a diverso concetto. ').

Simile alla mia è, poi l'analisi di Korn, nel suo Lehrbuch der Potentialtheorie ${ }^{3}$ ).

1) Sulle proprietà fondamentali della funzione potenziale nella immediata prossimità e nella estensione dell'agente. - Il Nuovo Cimento - (3) XXIII (1893).

${ }^{2}$ ) Intorno ad alcuni nuovi teoremi del sig. C. Neumann sulle funzioni poterziali. - Annali di Matematica - (2) X (1880).

$\left.{ }^{3}\right)$ Berlin, Dummler, (1899). 
Ora, com' è accennato da Beltrami, nel citato scritto, la funzione $k$, che figura in (1), ha, per se stessa, il significato di una funzione puramente data nei punti della superficie $\sigma-$ di una funzione, per fissare le idee, di una coppia di coordinate curvilinee $u, v$, atte a individuare $\mathrm{i}$ punti della superficie - per modo che, per discorrere di derivate della funzione $k$, rispetto alle coordinate cartesiane $a, b, c$ dei punti medesimi, bisogna concepirla proseguita nello spazio, ossia collegata con una funzione dei punti dello spazio, che si riduce ad essa, nei punti della superficie considerata. Ciò che a priori può farsi in infiniti modi.

Mi propongo, con questo breve scritto, di rilevare alcune circostanze generali, attinenti a simile collegamento, e di mostrarne l'applicazione ad espressioni delle derivate prime della funzione potenziale di doppio strato, e delle relative discontinuità, che sembrano meritevoli di qualche attenzione.

1. - Indichiamo, per un momento, con $\mathrm{F}(u, v)$ la funzione, intesa regolare, delle coordinate curvilinee ortogonali $u, v$, data nei punti della superficie $\sigma$; e con $f(x, y, z)$ indichiamo una funzione delle coordinate $x, y, z$ dei punti dello spazio, quale si voglia, purchè regolare, la quale si riduce a $\mathrm{F}(u, v)$ nei punti della superficie $\sigma$, cioè quando le $x, y, z$ si esprimano per mezzo delle $u, v$, conformemente alle equazioni parametriche della superficie $\sigma$.

Indichiamo poi con $\alpha_{1}, \rho_{1} \gamma_{1}$ e con $\alpha_{2}, \rho_{2}, \gamma_{2}$ i coseni di direzione della tangente alla linea $u$ e alla linea $v$, relative al punto $(x, y, z)$, prese con un senso convenuto, e con $\alpha_{3}, p_{3}, \gamma_{3}$ i coseni di direzione della normale $n$ nello stesso punto.

Ciò premesso, poniamo

$$
\left\{\begin{array}{l}
\frac{\partial f}{\partial x} \alpha_{1}+\frac{\partial f}{\partial y} \beta_{1}+\frac{\partial f}{\partial z} \gamma_{1}=\mathrm{U} \\
\frac{\partial f}{\partial x} \alpha_{2}+\frac{\partial f}{\partial y} \rho_{2}+\frac{\partial f}{\partial z} \gamma_{2}=\mathrm{V} \\
\frac{\partial f}{\partial x} \alpha_{3}+\frac{\partial f}{\partial y} \rho_{3}+\frac{\partial f}{\partial z} \gamma_{3}=\mathrm{W}
\end{array}\right.
$$


$\mathrm{U}$ e $\mathrm{V}$ hanno il significato di $\frac{\partial F}{\partial s_{u}}, \frac{\partial F}{\partial s_{v}}$, indicando, al solito, con $s_{u}$ e $s_{v}$ la misura dell'arco di linea $u$ e di linea $v$; c perciò sono invarianti per rispetto allo moltiplici forme possibili di $f$.

W ha, alla sua volta, il signficato di $\frac{\partial f}{\partial n}$; e rappresenta un elemento disponibile, in quanto è disponibile il collegamento di $\mathrm{F}(u, v)$ con una funzione dei punti dello spazio.

In particolare potremo supporre

$$
\mathrm{W}=0
$$

che si traduce in

$$
\frac{\partial f}{\partial n}=0
$$

Ciò torna immaginare, nei punti corrispondenti delle superficie parallele a $\sigma$, entro uno strato sottile a piacere, purchè finito, dalla parte verso cui volge $n$, mantenuto il valore di $f$ che appartiene ai punti di $\sigma$, ciò̀ $\mathrm{F}(u, v)$. Particolare collegamento, che, per brevità, shiameremo « normale».

Dalle (2) si ricava

$$
\left\{\begin{array}{l}
\frac{\partial f}{\partial x}=\alpha_{1} \mathrm{U}+\alpha_{2} \mathrm{~V}+\alpha_{3} \mathrm{~W} \\
\frac{\partial f}{\partial y}=\beta_{1} \mathrm{U}+\beta_{2} \mathrm{~V}+\beta_{3} \mathrm{~W} \\
\frac{\partial f}{\partial z}=\gamma_{1} \mathrm{U}+\gamma_{3} \mathrm{~V}+\gamma_{3} \mathrm{~W}
\end{array}\right.
$$

Con che, per ogni collegamento, si ha una particolaredeterminazione delle $\frac{\partial f}{\partial x}, \frac{\partial f}{\partial y}, \frac{\partial f}{\partial z}$, nei punti della superficie. 
Col collegamento normale, le (4) forniscono

(5)

$$
\frac{\partial f}{\partial x}=\alpha_{1} U+\alpha_{2} \mathrm{~V}, \frac{\partial f}{\partial y}=\beta_{1} \mathrm{U}+\beta_{2} \nabla, \frac{\partial f}{\partial z}=\gamma_{1} \mathrm{U}+\gamma_{2} \mathrm{~V} .
$$

2. - Richiamiamo ora le formule trovate per le derivate prime della funzione potenziale di doppio strato (1), nella ricordata Memoria. Cioè, cogli attuali simboli, mantenendo, del resto, ad $a, b, c$ e a $x, y, z$ il significato di coordınate del punto generico della superficie e del punto potenziato,

(6) $\frac{\partial f}{\partial x}=\int\left[\frac{\partial k}{\partial a} \frac{\partial \frac{1}{r}}{\partial n}-\left(\frac{\partial k}{\partial a} \frac{\partial \frac{1}{r}}{\partial a}+\frac{\partial k}{\partial b} \frac{\partial \frac{1}{r}}{\partial b}+\frac{\partial k}{\partial c} \frac{\partial \frac{1}{r}}{\partial c}\right) \alpha_{3}\right] d \sigma$

$$
+\int_{s} k\left(\frac{\partial \frac{1}{r}}{\partial b} d c-\frac{\partial \frac{1}{r}}{\partial c} d b\right)
$$

trasformabile in

$$
\begin{aligned}
& \frac{\partial \varphi}{\partial x}=\int_{\sigma} \frac{\partial k}{\partial a} \frac{\partial \frac{1}{r}}{\partial n} d \sigma+\frac{\partial}{\partial x} \int_{\sigma}^{\frac{\partial k}{\partial a} \alpha_{3}} \frac{\partial}{r} d \sigma+\frac{\partial k}{\partial y} \int_{\sigma}^{\frac{\partial b}{\alpha_{3}}} d \sigma+ \\
& +\frac{\partial}{\partial z} \int_{\sigma}^{\frac{\partial k}{\partial c} \alpha_{3}} \frac{\partial \frac{1}{r}}{r} d \sigma+\int_{s} k\left(\frac{\partial \frac{1}{r}}{\partial b} d c-\frac{1}{\partial c} d b\right)=
\end{aligned}
$$

donde, escludendo il caso dubbio che il punto limite $(x, y, z)$ appartenga al contorno $s$, si ricava

$$
\lim _{n>0} \frac{\partial \varphi}{\partial x}-\lim _{n<0} \frac{\partial \varphi}{\partial x}=4 \pi\left(\frac{\partial k}{\partial x}-\alpha_{3} \frac{\partial k}{\partial n}\right)
$$


Conformemente alle (4), abbiamo, tenendo calcoio del significato che acquistano attualmente $\mathrm{U}, \mathrm{V}, \mathrm{W}$,

$$
\frac{\partial k}{\partial a} \frac{\partial \frac{1}{r}}{\partial a}+\frac{\partial k}{\partial b} \frac{\partial \frac{1}{r}}{\partial b}+\frac{\partial k}{\partial c} \frac{\partial \frac{1}{r}}{\partial c}=\frac{\partial k}{\partial s_{u}} \frac{\partial \frac{1}{r}}{\partial s_{u}}+\frac{\partial k}{\partial s_{v}} \frac{\partial \frac{1}{r}}{\partial s_{v}}+\frac{\partial k}{\partial n} \frac{\partial \frac{1}{r}}{\partial n}
$$

donde

$$
(8)\left\{\begin{array}{c}
\frac{\partial k}{\partial a} \frac{\partial \frac{1}{\partial n}}{\partial n}-\left(\frac{\partial k}{\partial a} \frac{\partial \frac{1}{r}}{\partial a}+\frac{\partial k}{\partial b} \frac{\partial \frac{1}{v}}{\partial b}+\frac{\partial k}{\partial c} \frac{\partial \frac{1}{r}}{\partial c}\right) \alpha_{3} \\
=\left(\frac{\partial k}{\partial a}-\alpha_{3} \frac{\partial k}{\partial n}\right) \frac{\partial \frac{1}{r}}{\partial n}-\left(\frac{\partial k}{\partial s_{u}} \frac{\partial \frac{1}{\partial}}{\partial s_{u}}+\frac{\partial k}{\partial s_{v}} \frac{\partial \frac{1}{v}}{\partial s_{v}}\right) \alpha_{3} \\
=\left(\alpha_{4} \frac{\partial \frac{1}{\partial}}{\partial n}-\alpha_{3} \frac{\partial \frac{1}{\partial}}{\partial s_{u}}\right) \frac{\partial k}{\partial s_{u}}+\left(\alpha_{3} \frac{\partial \frac{1}{\partial n}}{\partial n}-\alpha_{3} \frac{\partial \frac{1}{\partial}}{\partial s_{v}}\right) \frac{\partial k}{\partial s_{v}}
\end{array} .\right.
$$

valendosi, conformemente alla prima delle (4), di

$$
\frac{\partial k}{\partial a}-\alpha_{3} \frac{\partial k}{\partial n}=\alpha_{1} \frac{\partial k}{\partial s_{u}}+\alpha_{2} \frac{\partial k}{\partial s_{v}} .
$$

Introducendo (8) in (6) e (9) in (7), si ottiene per $\frac{\partial \varphi}{\partial x}$, e per la relativa discontinuità, una forma, che si può chiamare intrinseca, perchè puramente formata colla funzione, data nei punti della superficie, che esprime il momento.

D'altra parte, valendosi del collegamento normale (\$ 2 ), la (7) acquista la forma semplicissima

$$
\lim _{n>0} \frac{\partial \varphi}{\partial x}-\lim _{n<0} \frac{\partial \varphi}{\partial x}=4 \pi \frac{\partial k}{\partial x}
$$


che fornisce

$$
\lim _{n>0} \frac{\partial \varphi}{\partial x}-\lim _{n<0} \frac{\partial \varphi}{\partial x}=0
$$

quando l'asse $x$ si supponga avere la direzione di $n$.

Noto infine che la deduzione della (9) dalla (6), dove sia introdotto il secondo membro della (8), si fa pure facilmente. Difatti, posto

$$
\begin{aligned}
& \int_{\sigma}\left(\frac{\partial k}{\partial a}-\alpha_{3} \frac{\partial k}{\partial n}\right) \frac{\partial \frac{1}{r}}{\partial n} d \sigma=\mathrm{I}_{n}, \int_{\sigma} \frac{\partial k}{\partial s_{u}} \alpha_{3} \frac{\partial \frac{1}{r}}{\partial s_{u}} d \sigma=\mathrm{I}_{u}, \\
& \int_{\sigma} \frac{\partial k}{\partial s_{v}} \alpha_{3} \frac{\partial \frac{1}{r}}{\partial s v} d \sigma=\mathrm{I}_{v}, \quad \int_{s} k\left(\frac{\partial \frac{1}{r}}{\partial b} d c-\frac{\partial \frac{1}{\partial}}{\partial c} d b\right)=\mathrm{I}_{s}
\end{aligned}
$$

si ha con ciò

$$
\frac{\partial \varphi}{\partial x}=I_{n}+I_{u}+I_{v}+I_{s}
$$

Ora, $\mathrm{I}_{n}$ fornisce l'indicata discontinuità, e la discontinuità di $I_{u}$ e di $I_{v}$ risulta nulla. Poichè si ha

$$
\mathrm{I}_{u}=-\frac{\partial}{\partial x} \int_{\sigma} \frac{\mathrm{K} \alpha_{1}}{r} d \sigma-\frac{\partial}{\partial y} \int_{\sigma} \frac{\mathrm{K} \beta_{1}}{r} d \sigma-\frac{\partial}{\partial z} \int_{\sigma} \frac{\mathbf{K} \boldsymbol{\gamma}_{1}}{r} d \sigma
$$

posto

$$
\frac{\partial k}{\partial s_{u}} \alpha_{8}=\mathrm{K}
$$

Con che la discontinuità di $I_{u}$ risulta

$$
4 \pi \mathrm{K}\left(\alpha_{1} \alpha_{3}+\beta_{1} \rho_{3}+\gamma_{1} \gamma_{3}\right)=0 \text {. }
$$

E allo stesso modo riesce nulla la discontinuità di $\mathrm{I}_{v}$. 
3. - A complemento della enunciata questione del collegamento di una funzione data nei punti di una superficie con una funzione dei punti dello spazio, rileviamo ancora che, in casi diversi da quello della funzione potenziale di doppio strato, precedentemente considerato, potrà riuscire assegnata la funzione $f(x, y, z)$ dei punti dello spazio, da cui, nell'ipotesi che i punti cadano sulla superficie, deve dedursi la funzione, $\mathrm{F}(u, v)$, di questi punti, che conviene al problema. Tale è il caso della discontinuità delle componenti dello spostamento elastico, ad una superficie, rappresentante un diaframma del campo (molteplicemente connesso) rappresentato dal corpo elastico, nelle "distorsioni di Volterra »'). In simili casi sono assegnate a priori, nei punti della superficie,

$$
\frac{\partial f}{\partial x}, \frac{\partial f}{\partial y}, \frac{\partial f}{\partial z},
$$

donde, per mezzo delle (2), risultano determinate U, V, W.

Pisa, settembre, 1917.

(') Cfr. la mia Nota Posizione e soluzione di alcune questioni attinenti all teoria delle distorsioni $e^{\prime}$ astiche. - Rendiconti della $R$. Accademia dei Lincei - (5) XXVI (1917). 